\title{
Using Holography to Evaluate CCD Camera Characteristics with High Accuracy
}

\author{
* E. Voelkl, ** D. Tang \\ * FEI Company, 5350 NE Dawson Creek Drive, Hillsboro, OR, 97124-5793 \\ ** FEI Company, Achtseweg Noord 5, 5651 GG Eindhoven, The Netherlands
}

Electron holography or specifically electron off-axis holography produces a two-beam-interference pattern with a well defined spatial frequency [1]. Using a CCD camera to record such a pattern results in pixel values that can be described in a scale free manner by:

$$
P_{m n} \sim 1+\mu \cos \left(2 \pi\left(m / s_{x}+n / s_{y}\right)+\varphi_{m n}\right)
$$

where $m, n$ are integer numbers with $0 \leq m<M, 0 \leq n<N$ and $M$ and $N$ are the number of pixels in $x$ - and $y$-direction of the CCD camera. Here we will assume $M=N$. Further, $s_{x}$ and $s_{y}$ are the sampling frequencies for the interference pattern along the $x$ - and $y$-axis of the CCD camera, $\mu$ is the (measured) fringe contrast, $P_{m n}$ is the actually measured value of the pixel at $(m, n)$ and $\varphi_{m n}$ is the lateral phase of the fringe pattern with respect to the CCD camera.

As reported in $[2,3]$, for such a data set the low bound of the resulting phase noise is determined as:

$$
\sigma_{\varphi} \geq \sqrt{2^{j}} \frac{2 R \sqrt{2 \pi} r}{\mu M \sqrt{\left\langle P_{m n}\right\rangle f\left(q_{x}, q_{y},\left\langle P_{m n}\right\rangle\right)}}
$$

where $j=0$ if no reference hologram was used for the reconstruction process (else: $\mathrm{j}=1$ ), $r$ is the radius (in pixels) of the basic reconstruction aperture ( 1 inside and 0 outside $r$ ), $R$ indicates the change in image size during the reconstruction process $(\mathrm{R}=1 / 2$ when going from a $1 \mathrm{k}$ by $1 \mathrm{k}$ image to a 512 by 512 image) and $\left\langle P_{m n}\right\rangle=\sum P_{m n} / M N$. The parameter $f$ is the transfer efficiency of the camera as described e.g., in [4] and depends on the sampling rate for the fringes as well as the average pixel count (for low pixel counts the electronic noise (dark current) can become a major contribution).

Given a digital off-axis hologram, the only unknown in eqn (2) is $f\left(q_{x}, q_{y},\left\langle P_{m n}\right\rangle\right)$. When evaluating holograms, it is advantageous to work with 2 holograms recorded consecutively as this allows suppression of artifacts from Fresnel fringes and distortions in the optical system as well as the fiber optic element of the CCD camera. By doing so, the transfer efficiency of a Gatan US1000 camera on a FEI Titan TEM is evaluated at $300 \mathrm{kV}$ for the case of a binning factor of 1 and 2 . Initial results for a binning factor of 2 , a sampling rate of $\left(s_{x}, s_{y}\right)=(5.92,9.14)$ and for $300 \mathrm{kV}$ electrons, showed a transfer efficiency of 0.47 .

The effect of $f\left(q_{x}, q_{y},\left\langle P_{m n}\right\rangle\right)$ is at least 2-fold and acts both as a modulation transfer function (MTF) and a detection efficiency (DE) factor [4,5]. The MTF has the effect of reducing the measured fringe contrast and can do so significantly. Its dampening effect usually increases with decreasing sampling rates. The DE is reasonably independent of the sampling rate but increases the noise in the 
reconstructed phase image just like the MTF. On the other hand, it does not affect the measured fringe contrast.

Because of the difference of the nature of the MTF and DE, $f$ may be re-written as:

$$
f\left(q_{x}, q_{y},\left\langle P_{m n}\right\rangle\right)=f^{M T F}\left(q_{x}, q_{y}\right) \cdot f^{D E}\left(\left\langle P_{m n}\right\rangle\right)
$$

where $f^{D E}$ acts directly as a weight factor for each $P_{m n}$ in the hologram, but $f^{M T F}$ acts directly as dampening factor for the fringe contrast $\mu$. By measuring the actual electron density versus the actual average pixel count for $\mu=0$ (a flat illumination), the main effect of the DE may be determined via $f^{D E}$. In this case, $f^{M T F}$ becomes a measurable quantity and allows deducing the true fringe contrast of the interference fringes by compensating for the effect of $f^{M T F}$.

Knowing the transfer efficiency of a camera can help regain the actual signal shape (not the noise), i.e., the signal before it was recorded. This can be of great interest for, e.g., high resolution transmission electron microscopy (HRTEM). Taking into account that $f^{M T F}$ can be further split into a scale dependent and scale independent part, it may be possible to evaluate and eventually design a dedicated holography CCD camera that is optimized for the transfer of information recorded with low sampling rates as is required for off-axis holography in both light- and electron optics.

\section{References}

[1] Introduction to Electron Holography, Kluwer Academic / Plenum Publishers, 1999

[2] E. Voelkl, Ultramicroscopy submitted.

[3] E. Voelkl et al., Microscopy Microanalysis 13(Suppl 2), 2007, p138-139.

[4] W.J. deRuijter, Micron 26 (1995)

[5] Image Science, J.C. Dainty and R. Shaw, Academic Press 1974 